\section{Mental health and wellbeing}

\section{OP61 UNDERSTANDING SOCIAL INEQUALITIES IN CHILD MENTAL HEALTH: FINDINGS FROM THE UK MILLENNIUM COHORT STUDY}

VSS Straatmann*, MC Campbell, CR Rutherford, SW Wickham, DTR Taylor-Robinson. Department of Public Health and Policy, University of Liverpool, Liverpool, UK

10.1136/jech-2017-SSMAbstracts.60

Background Child mental health is poor in the UK, with the most disadvantaged children experiencing worse outcomes and consequences over the course of their lives. Using a contemporary U.K. birth cohort, we therefore explored the social gradient in poor child mental health and the extent to which it is explained by other known risk factors for adverse child mental health outcomes.

Methods Analysis of the U.K. Millennium Cohort Study (MCS), based on 9818 children who participated in five survey sweeps (9 months, 5, 7 and 11 years old). The main outcome was child socio-emotional behavioural problems using the Total Difficulties Score of the Strengths and Difficulties Questionnaire (SDQ), at age 11. Relative Risk (RRs) and 95\% confidence intervals (CI) for socioemotional behavioural problems were estimated using Poisson regression, according to maternal education, which was used as a measure of socioeconomic circumstances at birth. Sequential models adjusted for risk factors for child mental health problems included demographic factors (sex, ethnicity and maternal age), family poverty, maternal mental health, and being bullied. Analyses were conducted using Stata/SE with svy commands to account for the sampling design and attrition.

Results By age 11, 10.4\% (95\%CI 9.6\%-11.2\%) of children had socioemotional behavioural problems. Children of mothers with no qualifications were more than five times as likely to have mental health problems compared to degree level (RR 5.4 [95\%CI 4.0-7.4]). Male sex, younger maternal age, poor maternal mental health, family poverty and being bullied, were all independently associated with an increased risk of child mental health problems. Adjusting for maternal mental health, family poverty and being bullied attenuated the RR for mental health problems in the lowest maternal education group compared to the highest $(4.2$ [95\%CI 3.0-5.9]); 4.0 (95\% CI 2.8-5.7); and 4.9 (95\% CI 3.5-6.8) respectively. Adjusting for all risk factors attenuated the RR to 3.4 (95\% CI 2.3-5.0).

Conclusion In a representative U.K. child cohort, we found one in ten children faced socioemotional behavioural problems at age 11. The risk was much greater in disadvantaged children. This was partially explained by the social patterning of maternal mental health, family poverty, and being bullied. The self-reported outcome is a limitation of this study. Future research should investigate critical/sensitive periods for these exposures over the life-course. Efforts to reduce inequalities in child mental health problems should focus on reducing socioeconomic inequalities and action on risk factors such as maternal mental health, child poverty, and bullying.

\section{OP62 WHAT ARE THE PHYSICAL AND PSYCHOLOGICAL HEALTH EFFECTS OF SUICIDE BEREAVEMENT ON FAMILY MEMBERS?: A QUALITATIVE STUDY}

\footnotetext{
${ }^{1,2} \mathrm{~A}$ Spillane ${ }^{*},{ }^{3} \mathrm{C}$ Larkin, ${ }^{1,2} \mathrm{P}$ Corcoran, ${ }^{1} \mathrm{~K}$ Matvienko-Sikar, ${ }^{1,2} \mathrm{E}$ Arensman. ${ }^{1}$ Department of Epidemiology and Public Health, University College Cork, Cork, Ireland; ${ }^{2}$ National Suicide Research Foundation, University College Cork, Cork, Ireland; ${ }^{3}$ Department of Emergency Medicine, University of Massachusetts Medical School, Worcester, USA
}

\subsection{6/jech-2017-SSMAbstracts.61}

Background Research indicates that experiencing the suicide of a relative can have a significant impact on family members' psychological health. However, research incorporating the impact of suicide bereavement on family members' physical health is sparse. Therefore, the aim of this study was to examine how family members have been physically and psychologically affected by a relative's suicide.

Methods This exploratory qualitative study is a follow-up to a larger case-control study, which examined the psychological, psychiatric and work-related factors associated with suicide in Ireland (SSIS-ACE, 2014-2017). Participants for the SSIS-ACE study were next-of-kin of persons who died by suicide or probable suicide, who were identified via coroner's records. All participants who completed the SSIS-ACE interview and who consented to further follow-up were invited by letter to take part in the current study. Semi-structured interviews, with the use of a topic guide were conducted with 18 relatives experiencing suicide bereavement. Eleven participants were female and seven were male; participant's ages ranged from 25-73 years. Duration of bereavement ranged from 15 to 37 months. Thematic analysis was used to analyse the data which was facilitated by the use of NVIVO 11 to organise the data. Results Preliminary findings indicate the emergence of five themes in two main domains, psychological and physical outcomes.

Psychological outcomes For most participants, the suicide was viewed as a predominantly negative turning point in their lives, where the death forced them to confront a new reality without their loved one. Secondly, immediate emotional reactions, including shock, disbelief, guilt, anger and surprise were reported. Suffering from persistent mental health difficulties was a recurrent theme among bereaved relatives: difficulties included depression, anxiety, stress, posttraumatic stress disorder, suicidal thoughts and suicide attempts. Coping mechanisms, both positive and negative, utilised by suicide survivors emerged as the fourth theme.

Physical outcomes Immediate physical reactions, including nausea, breathlessness, palpitations, chest pains and losing consciousness were physical reactions reported. Some of these physical conditions did not improve in the months after the death but rather persisted and sometimes worsened over time. Conclusion Family members bereaved by suicide are at risk of mental and physical health sequelae, while also being vulnerable to suicidal thoughts and suicide attempts. Participants were drawn from a small geographic area and the findings of this study may not be generalisable to other settings. From a policy perspective, this study highlights the importance of providing support services for this group following suicide bereavement. 